Virtual Mentor. August 2004, Volume 6, Number 8.

doi: 10.1001/virtualmentor.2004.6.8.ccas3-0408

Clinical Cases

\title{
Is Chronic Pain Treatment the Neurologist's Job?
}

\section{Patients who are seeking pain management with opioids can be difficult for neurologists to manage.}

\author{
Commentary by Erich Garland, MD
}

Dr. Fields has been a neurologist for 17 years. He has seen much improvement in pain management—particularly as part of palliative care at the end of life-over the course of his practice. Many challenges remain, however. Pain from inoperable lesions and pain that persists after surgery can be impossible to treat successfully. Patients with such pain can be in tremendous distress — often unable to continue at their jobs and sometimes unable even to rest without drugs that affect awareness and have other serious side effects.

One day a colleague of Dr. Fields, an orthopedic surgeon named David Burns, asks whether Dr. Fields will consent to see a patient of his. Dr. Burns relates an abbreviated history of his patient, Eleanor Mitchell, now 47 years old. Nearly 3 years ago, Mrs. Mitchell lost her balance while helping her husband carry a new air conditioning unit up the front stairs to their house. She experienced immediate lumbar pain. She called Dr. Burns who, after examination, prescribed a muscle relaxant and bed rest. Two weeks later, Mrs. Mitchell returned, still in pain. A subsequent lumbosacral MRI showed herniation of Mrs. Mitchell's L5-S1 disc with a small fragment compressing the left S1 root.

When Dr. Burns recommended surgery, Mrs. Mitchell rejected the idea. She had heard about mistakes in back surgery, she said. She also knew of someone her husband worked with who had injured his back at work and had as much pain after back surgery as before. He had to go on permanent disability. Mrs. Mitchell didn't want that. She decided instead to try physical therapy and "painkillers" and see how it went.

After 18 months, Mrs. Mitchell asked to be scheduled for surgery. She had missed a good deal of work and had been in moderate to severe pain "every minute of the day" except when sleeping-and getting to sleep wasn't so easy either. Dr. Burns performed surgery to remove the fragments and relieve the compression, and Mrs. Mitchell recovered without complication. After the appropriate recovery period, however, her pain persisted. In fact, it became more intense. She complained of pain in her left leg also. Upon Dr. Burns' recommendation, Mrs. Mitchell consulted another orthopedist and a neurosurgeon, both of whom found no other surgical indications. Now Dr. Burns was turning to his neurology colleague, Dr. Fields.

"She's had PT on and off without success and attended a pain clinic briefly but found that sympathetic and regional nerve blocks provided only transient-if any-relief. I think this is really more of a neurology case than an orthopedic case at this point," Dr. Burns said.

Dr. Fields, however, was reluctant to accept another patient with chronic pain.

"I have much sympathy for Mrs. Mitchell and others with her sort of intractable pain. You know I do, Dave. But it seems that all treatment doors have been opened and closed. She doesn't need a neurologist to write her a script every month for the rest of her life," Dr. Fields said. "Her internist can do that. Besides, I have quite a few patients who are being maintained on heavy-duty opioids, and I have to watch that. I could be escorted out of here in handcuffs some day. It's happened. Especially if the patient appears to be doctor-hopping," Dr. Fields said. 


\section{Commentary}

Treatment of chronic pain is both an art and science. It requires that the treating physician have an understanding of the pathophysiology of pain and a history of treatments that have failed and those that have provided some relief. Physicians have traditionally been trained to diagnose and treat disease as the cause of pain, but few have the training to treat chronic pain effectively. In this case study the patient tried conservative management for 18 months before choosing surgery as an option. Postoperatively her pain persisted and eventually worsened. Although she consulted 2 other surgeons for opinions and attended a pain clinic, she continued to experience intractable pain.

Before accepting this patient, I would want to know why PT and a pain clinic could not provide Mrs. Mitchell adequate relief of her pain. Review of her medical records would be the most appropriate first step. Then I would offer Mrs. Mitchell an appointment to get a medical history, psychosocial history, and physical exam and determine what her expectations are before agreeing to provide long-tem opioid management. After a thorough history, physical exam, and psychosocial history, a diagnosis should be made, and all prior treatments and diagnostic tests verified; treating physicians and pharmacies used should also be confirmed. Goals for treatment such as return to work, less pain, and improved activities should be documented in the medical record. Treatment should be monitored and drug testing done when suspicion of inappropriate use arises. Patients must be informed about the side effects associated with chronic opioid use, and their informed consent must be obtained. One physician and one pharmacy must be chosen for the opioid prescriptions. A written contract is the best way to document this process. Patients receiving opioids should be seen by the physician on a regular schedule. The physician should prescribe long-acting opioids on a time schedule, document treatment outcomes in the medical record, and reevaluate the benefits and risks of continued opioid use periodically. Taking these steps will alleviate the concerns Dr. Biels expresses in his reluctance to take on the care of Mrs. Mitchell.

Opioids are not the only treatment option. Optimal pain management also includes physical conditioning, non-opioid medications, and coping skills to obtain the best quality of life from the treatment plan. Recovery from chronic pain requires educating the patient for a life without pain. This can entail major psychological adjustment to overcome fear, anger, shame, loss of self-esteem, loss of respect, and codependency. Finally, psychiatric disorders are a common comorbidity with chronic pain, a fact that makes treatment difficult and non-rewarding for physicians to manage.

Erich Garland, MD, is a board-certified neurologist who is medical director of Idaho Falls Neurology, Idaho.

The people and events in this case are fictional. Resemblance to real events or to names of people, living or dead, is entirely coincidental. The viewpoints expressed on this site are those of the authors and do not necessarily reflect the views and policies of the AMA.

(C) 2004 American Medical Association. All Rights Reserved. 